\title{
Future facilities for solar and stellar activity research
}

\author{
Klaus G. Strassmeier \\ AIP, Astrophysical Institute Potsdam, D-14482 Potsdam, Germany \\ email: kstrassmeier@aip.de
}

\begin{abstract}
I try to list the currently ongoing instrumental activities for solar and stellar activity research. Only projects that lead to operational ground-based facilities no later than 2013 and to operational space-based observatories no later than 2016 are considered. Any facility already in operation or any instrument under construction but with a very wide range of usage is excluded from this listing (like e.g. ALMA, Herschel or SOFIA). No details on science programs are given. The text is organized according to space, radio, and optical/IR projects.
\end{abstract}

Keywords. telescopes, space vehicles, instrumentation: miscellaneous

SOLAR-B (now named Hinode) has just been launched (Sept. 22, 2006).

CoRoT is up for launch late November 2006. Its science mission is twofold. Firstly, it will focus on ultra-high precision photometry of very bright stars for asteroseismology of all kinds of non-radially pulsating stars. Secondly, it will perform photometry of some 30,000 stars down to 15th magnitude to search for transits of extrasolar planets.

SDO (Solar Dynamic Observator) is getting ready for launch in 2008. It is the anticipated $\mathrm{SOHO}$ successor and will employ, besides other instruments, the Helioseismic Magnetic Imager which will allow a full inversion of the internal solar magnetic field.

Kepler is planned for launch in early 2009. Its aim is to detect earth-sized planets from a massive transit search in a dedicated 100-sqr degree field in Cygnus with $>100,000$ stars. It employs high precision integral-light photometry over the period of $4 \mathrm{yr}$.

Gaia is one of the ESA cornerstone missions planned for launch in 2012. Its aim is to measure positions, parallaxes and proper motions of up to 1 billion stars with precisions of up to $\mu$-arcseconds. 7 -channel photometry and $\mathrm{km} / \mathrm{s}$-level radial velocities of a smaller subsample are also among its output. Final data are expected no earlier than 2016.

WSO-HIRDES (World Space Observatory - HIgh Resolution Double Echelle spectrograph) is a mission concept for possible launch in 2016 or later. It is based on a $1.7 \mathrm{~m} \mathrm{UV}$ optimized telescope with a double spectrograph for the wavelength range $103-310 \mathrm{~nm}$ at a resolution of 50,000 .

ATA (Allen Telescope Array) is a $0.5-11.2 \mathrm{GHz}$ array of 350 (final configuration) $6 \mathrm{~m}$ dishes at Hat Creek, California. Its FOV is $2.45^{\circ}$ at $21 \mathrm{~cm}$. Operated by the SETI Institute it will monitor 1 million stars in the $1-10 \mathrm{GHz}$ range and some 40 billion stars at $1.450 \mathrm{GHz}$. It could provide the most comprehensive view of the galactic magnetic field.

LOFAR (Low Frequency Array) is a digital new-technology ground-based radio spectrointerferometer for the $10-240 \mathrm{MHz}$ range. In its final configuration in 2009 it consists of dipole antenna fields across northern Europe, mainly in the Netherlands. The array observes the visible sky all the time, pointings will be done posteriori.

STELLA (Stellar Activity) is a fully robotic facility at the Teide Observatory, Tenerife. It consists of two $1.2 \mathrm{~m}$ telescopes that feed a high-resolution echelle spectrograph (390 $870 \mathrm{~nm}, R=55,000)$ and a wide-field imaging photometer $\left(22^{\prime} \times 22^{\prime}, 0.3^{\prime \prime} / \mathrm{px}, 17\right.$ filters $)$, respectively. It will be fully operational by 2007 . 
APF (Automated Planet Finder). A $2.4 \mathrm{~m}$ automated telescope currently under construction at Lick Observatory. Its sole instrument is a 'planetometer' designed for $1 \mathrm{~m} / \mathrm{s}$ precision radial velocity measurement based on an iodine cell.

Pan-STARRS (Panoramic Survey Telescope and Rapid Response System) will consist of four $1.8 \mathrm{~m}$ telescopes on Haleakala, Maui, Hawaii. The first telescope is already being commissioned. All four telescopes are wide-field imaging systems with a 1.4-Gigapixel CCD camera each (based on $64600 \times 600$ orthogonal-transfer CCD arrays). Its main scientific goal is to search for Near Earth Objects.

DCT (Discovery Channel Telescope). Installed and operated by Lowell Observatory in Arizona by 2012, the $4.2 \mathrm{~m}$ DCT will provide wide-field prime-focus imaging with a scientific focus towards faint Kuiper-belt objects.

LAMOST (Large-sky Area Multi-Object fibre Spectroscopy Telescope) is a $4 \mathrm{~m}$ segmented mirror telescope for the Xinglong station in China for 2009. 4000 fibers feed stellar light from a 5-degree FOV to 16 spectrographs covering a wavelength range of $370900 \mathrm{~nm}$ at resolutions of $R=1-5,000$ and $5-10,000$, respectively.

LBT (Large Binocular Telescope) consists of two $8.4 \mathrm{~m}$ mirrors on a joint mount with altogether 6 foci per telescope, two of which are interferometric. One of its instruments is PEPSI, the Potsdam Echelle Polarimetric and spectroscopic Instrument. It will provide full Stokes-vector spectropolarimetry at spectral resolution 120,000 for the wavelength range $450-1050 \mathrm{~nm}$. Its integral light modus enables resolving powers of 40,000 and 300,000 for the $390-1050 \mathrm{~nm}$ range. Located on Mt Graham in Arizona, the LBT and PEPSI will go into routine operation in 2009.

LSST (Large Synoptic Survey Telescope). Built upon the LBT mirror experience, the LSST is a $8.4 \mathrm{~m}$ ultra-wide field telescope with a FOV of $3.5^{\circ}$. Its three-reflection design provides a $64 \mathrm{~cm}$ diameter focal plane that will be paved with $2004 \mathrm{k} \times 4 \mathrm{k}$ CCDs with altogether $3.2 \mathrm{Gpx}$. It is expected to provide a full image of the visible sky every night. The telescope is expected to be located at Cerro Pachon in Chile by 2013 .

GREGOR is the new German solar telescope on Tenerife with first light in 2007. With an aperture of $1.5 \mathrm{~m}$ and integrated multi-conjugate adaptive optics (at second light) it is designed for high spatial resolution spectropolarimetry for the optical and IR range (up to $10 \mu \mathrm{m}$ ). Its night time is dedicated to the solar-stellar connection.

ATST (Advanced Technology Solar Telescope). The next-generation solar telescope to be build on Maui, Hawaii around 2012. A $4 \mathrm{~m}$ off-axis parabolic mirror will enable unprecedented image quality and shall provide enough light for high time resolution IR spectropolarimetry.

CONCORDIA (the French-Italian Antarctic station at Dome C). Located at $3280 \mathrm{~m}$ on the east Antarctic plateau with an average night temperature of $-63^{\circ}$ and practically zero (absolute) humidity, Dome $\mathrm{C}$ is 'between Earth and Space'. A number of astrophysics projects are foreseen for the years 2008-2013. Among them are IRAIT (a $80 \mathrm{~cm}$ JHKLM imaging telescope for 2008), A-STEP (a $40 \mathrm{~cm}$ optical telescope for planet transit photometry for 2008), ICE-T (a dual $60 \mathrm{~cm}$ ultra-wide-field ultra-high-precision optical photometer for 2011), and PILOT (a $2.4 \mathrm{~m}$ IR and optical high-resolution imager for 2013).

\section{Acknowledgements}

Contributions to this talk came from my AIP colleagues M.I. Andersen, G. Mann, M. Weber and M. Woche, and Xiangqun Cui (NAO, China), A. Kosovichev (Stanford), S. Vogt (Lick) and R. Kudritzki (Hawaii) to whom I am thankful. I also acknowledge the many www sites that provide the pictorial information beyond the mouth-to-ear approach. 